\title{
Facile synthesis of chitosan-capped ZnS quantum dots as an eco-friendly fluorescence sensor for rapid determination of bisphenol $A$ in water and plastic samples $\dagger$
}

\author{
Xianyi Cao, $\$$ Fei Shen, $\$$ Minwei Zhang, Jiaxin Bie, Xin Liu, Yeli Luo, Jiajia Guo \\ and Chunyan Sun*
}

\begin{abstract}
This paper describes a novel eco-friendly fluorescence sensor for determination of bisphenol A (BPA) based on chitosan-capped ZnS quantum dots (QDs). By using safe and inexpensive materials, nontoxic ZnS QDs were synthesized via an environment-friendly method using chitosan as a capping agent. The as-prepared ZnS QDs exhibited characteristic absorption (absorbance edge at $310 \mathrm{~nm}$ ) and emission (maxima at $430 \mathrm{~nm}$ ) spectra with a relatively high fluorescence quantum yield of $11.8 \%$. Quantitative detection of BPA was developed based on fluorescence quenching of chitosan-capped ZnS QDs with high sensitivity and selectivity. Under optimal conditions, the fluorescence response of ZnS QDs was linearly proportional to BPA concentration over a wide range from 0.50 to $300 \mu \mathrm{g} \mathrm{L}^{-1}$ with a detection limit of $0.08 \mu \mathrm{g} \mathrm{L}^{-1}$. Most of the potentially coexisting substances did not interfere with the BPA-induced quenching effect. The proposed analytical method for BPA was successfully applied to water and plastic real samples. The possible quenching mechanism is also discussed.
\end{abstract}

Received 21st December 2013

Accepted 17th March 2014

DOI: $10.1039 / \mathrm{c} 3 \mathrm{ra} 47868 \mathrm{~h}$

www.rsc.org/advances males, the increasing rates of breast cancer, the increase in other diseases linked to endocrine dyscrasia, as well as the increase in neural and behavioral changes in infants and children. ${ }^{6,7}$ Thus there is an urgent need to monitor the presence of BPA in daily water correlated to BPA-based food contact materials.

Traditional detection methods for BPA, such as highperformance liquid chromatography (HPLC), ${ }^{8}$ liquid chromatography/mass spectrometry (LC/MS), ${ }^{9}$ gas chromatography/ mass spectrometry (GC/MS), ${ }^{10}$ enzyme-linked immunosorbent assay (ELISA) ${ }^{\mathbf{1 1}}$ and capillary electrophoresis (CE), ${ }^{12}$ require expensive instruments, considerable time, experienced technicians and complex sample pretreatment, so their application in on-site rapid analysis is extremely limited. Recently, different types of analytical methods, including electrochemistry, ${ }^{\mathbf{1 3}}$ chemiluminescence, ${ }^{\mathbf{1 4}}$ quartz crystal microbalances (QCM) ${ }^{15}$ aptasensor-based colorimetry with $\mathrm{Au}$ nanoparticles, ${ }^{\mathbf{1 6}}$ surface plasmon resonance (SPR) biosensor, ${ }^{17}$ liposome chromatography ${ }^{18}$ etc. , have been developed to determine BPA, but most of them suffer from complex chemical synthesis, the use of volatile organic solvents, high cost or poor sensitivity. Therefore, developing rapid, simple, sensitive, low-cost and eco-friendly methods for BPA detection has become essential.

Fluorescence assay is a promising analytical technique with the advantages of requiring less sample and having low cost, high sensitivity, a quick response and easy operation. Thus, in recent years, it has been widely used in biochemical, food and
Department of Food Quality and Safety, College of Quartermaster Technology, Jilin University, Changchun 130062, China. E-mail: sunchunyan1977@163.com; sunchuny@jlu.edu.cn; Fax: +86 431 87836391; Tel: +86 43187836375

$\dagger$ Electronic supplementary information (ESI) available. See DOI: $10.1039 / \mathrm{c} 3 \mathrm{ra} 47868 \mathrm{~h}$

\$ The first two authors contributed equally to this work. 
environmental science. There have been several reports on fluorescence determination for $\mathrm{BPA},{ }^{19-21}$ and the detection limits are comparable with those of HPLC, ${ }^{8}$ LC/MS ${ }^{9}$ and GC/ MS, ${ }^{10}$ and are approximately 1-3 orders of magnitude lower than those reported by other methods such as ELISA, ${ }^{11} \mathrm{CE},{ }^{\mathbf{1 2}}$ electrochemistry ${ }^{\mathbf{1 3}}$ and chemiluminescence. ${ }^{\mathbf{1 4}}$ BPA can give a fluorescence signal by itself as its molecule possesses a conjugated cyclic structure, but in aqueous solution the signal is so weak that direct determination has poor detection performance. ${ }^{22}$ Some fluorescent probes are utilized for detection of BPA, such as fluorescent dyes, ${ }^{19} \mathrm{CdSe}^{20}$ and $\mathrm{CdTe}^{21}$ quantum dots (QDs). However, most fluorescent dyes have the

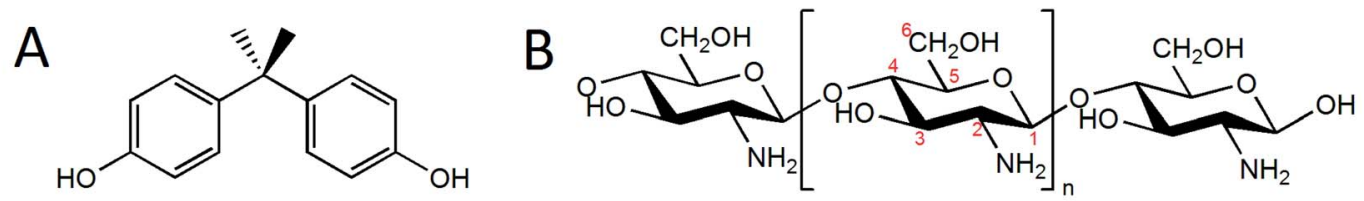
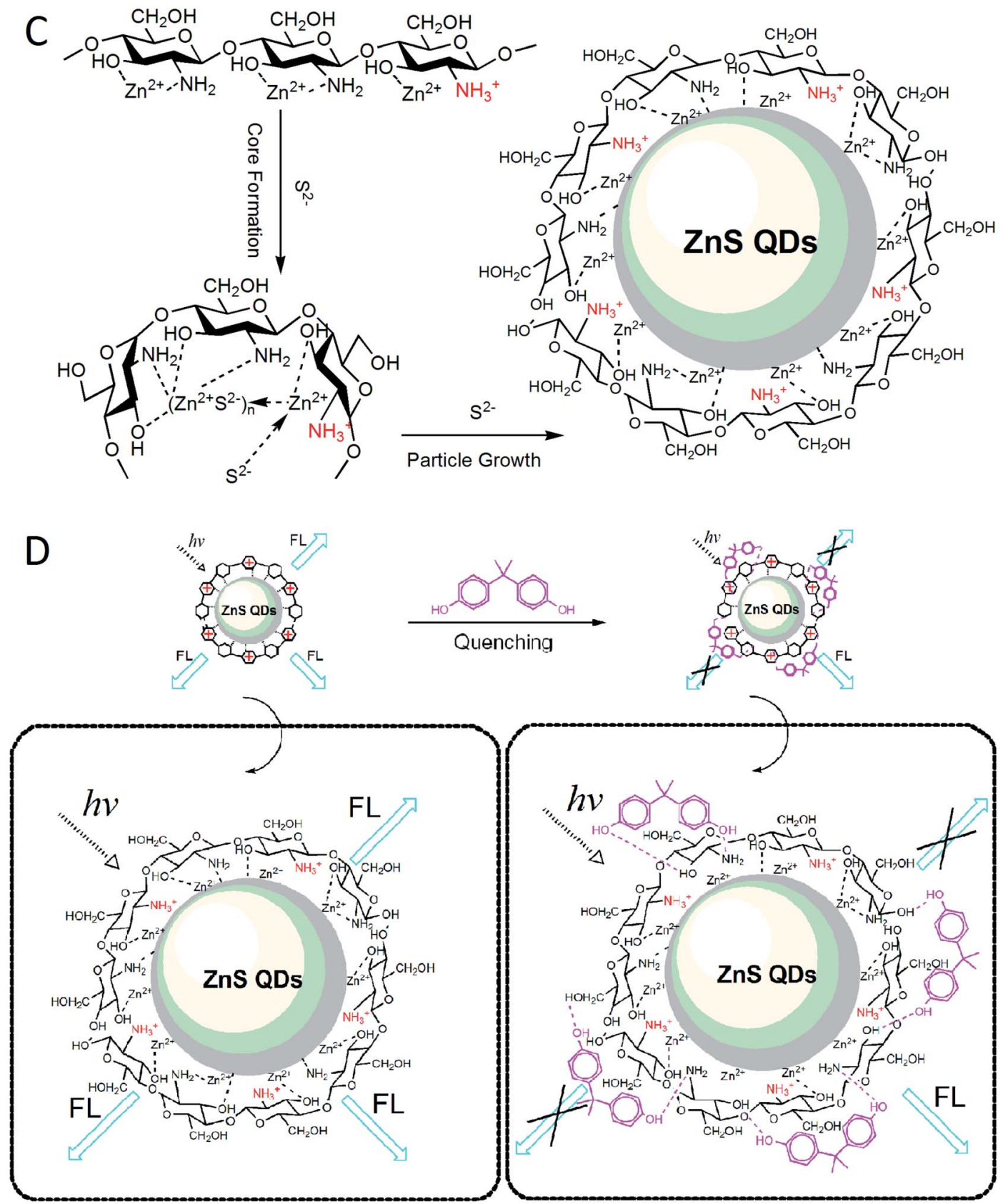

Scheme 1 (A) The molecular structure of BPA. (B) The molecular structure of chitosan. (C) Formation process of chitosan-capped ZnS QDs. (D) The proposed mechanism for the quenching effect of BPA on the fluorescence of chitosan-capped ZnS QDs. 
disadvantages of a low photobleaching threshold and poor chemical stability and biocompatibility, and $\mathrm{Cd} / \mathrm{Se} / \mathrm{Te}-$ based QDs have been confirmed to have strong cytotoxic activity. ${ }^{23}$ Thus, Zn-based QDs may be regarded as a promising new choice of nanophosphors for detection of BPA, as they are a type of nontoxic (or low-toxic) QDs compared with traditional $\mathrm{Cd} / \mathrm{Se} /$ Te-based QDs. ${ }^{24}$ To the best of our knowledge, there are no reports on fluorescence detection for BPA with $\mathrm{Zn}$-based QDs. Chitosan, $\beta$-(1,4)-2-amino-2-deoxy-D-glucose, is a unique cationic biocompatible polysaccharide built by repeating units of $\mathrm{N}$-acetyl-D-glucosamine and D-glucosamine, derived from the partial deacetylation of chitin, a natural polysaccharide extracted from crustacean shells. Chitosan has a desirable chelating ability with transition metal ions due to its special structure, which makes it possible for its metal ion complexes to be used as precursors to synthesize QDs. Moreover, its high viscosity can effectively prevent the aggregation of QDs, which greatly enhanced the stability of QDs in aqueous solution. ${ }^{25}$

In this paper we present a novel eco-friendly fluorescence sensor for detection of BPA based on chitosan-capped ZnS QDs. Chitosan was used as modifier as well as stabilizer, and welldispersed ZnS QDs with a uniform size were synthesized. By controlling the reaction conditions, the fluorescent properties of chitosan-capped ZnS QDs could be well regulated. The obtained water-soluble QDs were around $1.8 \mathrm{~nm}$ in diameter and displayed excellent fluorescent properties. We investigated the interaction between chitosan-capped ZnS QDs and BPA in aqueous solution. It was found that BPA dramatically quenched the fluorescence of chitosan-capped ZnS QDs, and the change of fluorescence intensity was proportional to the concentration of BPA. Based on this phenomenon, a sensitive, simple, rapid, lowcost and environment-friendly fluorescence method has been established for detection of BPA. Interference tests showed that some potentially co-existing substances, such as common inorganic ions and natural small molecules, have little interference. The possible mechanism of the proposed sensing method for BPA is also discussed (Scheme 1). This green method has been applied to the determination of BPA content in water and plastic samples and satisfactory results have been obtained.

\section{Experimental}

\subsection{Chemicals, materials and apparatus}

All chemicals were of analytical grade and used as received without further purification. High purity nitrogen (99.999\%) and double deionized water (DDW) were used in all experiments. Zinc acetate $\left[\mathrm{Zn}\left(\mathrm{CH}_{3} \mathrm{COO}\right)_{2} \cdot 2 \mathrm{H}_{2} \mathrm{O}\right]$, sodium sulfide $\left(\mathrm{Na}_{2} \mathrm{~S} \cdot 9 \mathrm{H}_{2} \mathrm{O}\right)$ and acetic acid were purchased from Xilong Chemical Co., Ltd (Shantou, Guangdong, China). Chitosan (high molecular weight, $\geq 75 \%$ deacetylated) and BPA were purchased from Sigma-Aldrich (St. Louis, Missouri, USA). $\mathrm{CaCl}_{2}$, $\mathrm{NaCl}, \mathrm{NH}_{4} \mathrm{Cl}, \mathrm{MgCl}_{2}, \mathrm{MnCl}_{2}, \mathrm{CuCl}_{2}, \mathrm{AlCl}_{3}, \mathrm{FeCl}_{3}, \mathrm{ZnCl}_{2}, \mathrm{BaCl}_{2}$, $\mathrm{KNO}_{3}, \mathrm{Na}_{3} \mathrm{PO}_{4}, \mathrm{Na}_{2} \mathrm{SO}_{4}, \mathrm{Na}_{2} \mathrm{CO}_{3}, \mathrm{AgNO}_{3}, \mathrm{Hg}\left(\mathrm{NO}_{3}\right)_{2}, \mathrm{NaOH}$, $\mathrm{CH}_{3} \mathrm{COONa}, \mathrm{HCl}$, glucose, lactose and glycine were purchased from Beijing Chemical Reagent Company (Beijing, China). Rhodamine 6G (Rh 6G) was obtained from Sinopharm
Chemical Reagent Co., Ltd (Shanghai, China). Different brands of packaged drinking water, plastic cups, feeding bottles, microwave lunch boxes and epoxy resin-based bowls were procured from a local market. Tap and rain water were collected in our laboratory and campus, respectively.

The absorption spectra were recorded on a $2550 \mathrm{UV}$-vis spectrophotometer (Shimadzu, Tokyo, Japan). The fluorescence spectra were obtained on an RF-5301PC fluorescence spectrophotometer (Shimadzu, Tokyo, Japan) with both the exciting and emission slits set at $5 \mathrm{~nm}$. High resolution transmission electron microscopy (HRTEM) measurements were made on a TECNAI F20 (FEI Co., Eindhoven, Netherlands) operated at an accelerating voltage of $200 \mathrm{kV}$. A drop of the QDs solution was drop-cast on ultra-thin carbon film-supported copper grids and subsequently air-dried before HRTEM analysis. FT-IR spectra were recorded with an IRPrestige-21 FT-IR spectrometer (Shimadzu, Tokyo, Japan). Zeta potential was obtained on a Zetasizer Nano ZS90 particle size analyzer (Malvern, Worcestershire, UK). The ultrasonic treatment was carried out on a $125 \mathrm{KQ}-$ 300DE ultrasonicator (Kunshan, Shanghai, China). The centrifugation was performed on a CR20B2 refrigerated centrifuge (Hitachi, Tokyo, Japan). Magnetic stirring was carried out with a GL-3250B magnetic stirrer (Qilinbeier, Haimen, China). All pH measurements were carried out with a Model pHS-3C pH meter (Chenghua, Shanghai, China). All optical measurements were performed at room temperature under ambient conditions.

\subsection{Synthesis and purification of water-soluble chitosan- capped ZnS QDs}

Chitosan is nearly insoluble in strong acidic and neutral media but soluble in weak acidic media, as the amine group is protonated to $\mathrm{NH}_{3}{ }^{+}{ }^{26}$ thus we used $1 \%$ (v/v) acetic acid aqueous solution to dissolve chitosan. Water-soluble chitosan-capped ZnS QDs were synthesized according to the procedure described previously with some modification. ${ }^{26,27} 0.22 \mathrm{~g}$ of $\mathrm{Zn}\left(\mathrm{CH}_{3} \mathrm{COO}\right)_{2} \cdot 2 \mathrm{H}_{2} \mathrm{O}$ was firstly added to $99 \mathrm{~mL}$ of $0.05 \%(\mathrm{w} / \mathrm{v})$ chitosan solution under constant stirring and heated at $80{ }^{\circ} \mathrm{C}$ for $20 \mathrm{~min}$ to facilitate a chelating balance. After the solution naturally cooled down to room temperature, $0.24 \mathrm{~g}$ of $\mathrm{Na}_{2} \mathrm{~S} \cdot 9 \mathrm{H}_{2} \mathrm{O}$ was dissolved in $1 \mathrm{~mL}$ of ice water and then added dropwise to the solution in an ice bath under continuous stirring and protection of $\mathrm{N}_{2}$. The molar ratio of $\mathrm{Zn}^{2+}: \mathrm{S}^{2-}$ was $1.5: 1$. The addition of $\mathrm{Na}_{2} \mathrm{~S}$ resulted in the formation of a milky white suspension immediately, and the solution was constantly stirred for $100 \mathrm{~min}$. Then the solution containing chitosan-capped ZnS QDs was centrifuged at 12000 rpm for $10 \mathrm{~min}$. The precipitated particles were washed three times using DDW and $0.1 \%(\mathrm{v} / \mathrm{v})$ acetic acid aqueous solution respectively to remove the adhered impurities and excess chitosan. The washed chitosan-capped ZnS QDs were dried in a vacuum oven at $60{ }^{\circ} \mathrm{C}$ for $24 \mathrm{~h}$. Finally, the prepared QDs were dispersed in $100 \mathrm{~mL} \mathrm{1 \%} \mathrm{(v/v)} \mathrm{acetic} \mathrm{acid} \mathrm{aqueous} \mathrm{solution} \mathrm{again}$ and stored in a refrigerator at $4{ }^{\circ} \mathrm{C}$ for further use. The QDs suspension can remain clear without any sedimentation for months.

To obtain high-quality chitosan-capped ZnS QDs, the influences of different synthesis conditions including the 
concentration of $\mathrm{Zn}^{2+}$ and chitosan, the molar ratio of $\mathrm{Zn}^{2+}: \mathrm{S}^{2-}$ and the reaction time on the fluorescence intensity of ZnS QDs were investigated. According to the evaluation for the above conditions, the concentration of $\mathrm{Zn}^{2+}$ and chitosan, the molar ratio of $\mathrm{Zn}^{2+}: \mathrm{S}^{2-}$ and the reaction time were optimized to be $1.0 \times 10^{-4} \mathrm{~mol} \mathrm{~L}^{-1}, 0.50 \mathrm{~g} \mathrm{~L}^{-1}, 1.5: 1$ and $100 \mathrm{~min}$, respectively. Under these synthesis conditions, the as-prepared ZnS QDs can exhibit relatively high fluorescence emission intensity and good analytical performance for detection of BPA.

\subsection{Analytical procedure}

Typically, in a $5 \mathrm{~mL}$ test tube, a certain volume of $500 \mu \mathrm{g} \mathrm{L} \mathrm{L}^{-1} \mathrm{BPA}$ aqueous solution was added and the solution was diluted to $2 \mathrm{~mL}$ with DDW. Then the solution was mixed with $1 \mathrm{~mL}$ of $3.5 \times 10^{-6} \mathrm{~mol} \mathrm{~L}^{-1}$ chitosan-capped ZnS QDs. After the mixture was homogenized thoroughly and equilibrated for $4.0 \mathrm{~min}$ at room temperature, the fluorescent intensity was recorded at an excitation wavelength of $315 \mathrm{~nm}$. The calibration curve for BPA was established according to the ratio of fluorescence intensity, that is, $F_{0} / F$, where $F_{0}$ and $F$ are the maximum emission intensities of ZnS QDs in the absence and presence of certain concentrations of BPA, respectively.

\subsection{Detection of BPA in real samples}

Tap and packaged drinking water were directly determined for the presence of BPA without any pretreatment. Rain water was filtered with a $0.45 \mu \mathrm{m}$-filter membrane to remove particulate matter.

According to Chinese National Standard GB/T 23296.1-2009 (Materials and articles in contact with foodstuffs - Plastics substances subject to limitation: Guide to test methods for the specific migration of substances from plastics to foods and food simulants and the determination of substances in plastics and the selection of conditions of exposure to food simulants), the contact temperature and time for the specific migrant test of BPA from plastic cup and feeding bottle to water-based food or food simulant should be chosen as $100{ }^{\circ} \mathrm{C}$ (or reflux temperature) and $240 \mathrm{~min}$, respectively. In order to shorten the pretreatment time for rapid detection of these plastic samples, ultrasonic and microwave extraction were respectively chosen and applied in our experiments according to the actual usages of the samples. An ultrasonicator $(220 \mathrm{~V}, 200 \mathrm{~W})$ and a microwave oven $(220 \mathrm{~V}, 500 \mathrm{~W})$ were used in the extraction procedures. Plastic cups, feeding bottles, microwave lunch boxes and epoxy resin-based bowls were washed with DDW thoroughly, solarized and cut into small fragments about $5 \mathrm{~mm} \times 5 \mathrm{~mm}$ size. Next, $10 \mathrm{~g}( \pm 0.0001 \mathrm{~g})$ of each plastic sample was put into a conical beaker and $100 \mathrm{~mL}$ of DDW was added. The solutions containing plastic cup or feeding bottle fragments were ultrasonic-extracted for $90 \mathrm{~min}$ in a water bath $\left(90 \pm 0.5{ }^{\circ} \mathrm{C}\right)$. The solutions containing microwave lunch box or epoxy resin-based bowl fragments were microwave-heated for $10 \mathrm{~min}$. Then all the sample leaching solutions were cooled to room temperature, filtered with a $0.45 \mu \mathrm{m}$-filter membrane and rediluted to $100 \mathrm{~mL}$ with DDW. Finally, $1 \mathrm{~mL}$ of the water or plastic leaching solution samples was used for BPA determination according to the proposed method in Section 2.3. In order to investigate the recoveries of these water and plastic leaching solution samples, a certain amount of BPA was doped into these samples, which were then pretreated and analyzed in accordance with the above procedure.

\section{Results and discussion}

\subsection{Characteristics of chitosan-capped ZnS QDs}

Due to the protonation of the $-\mathrm{NH}_{2}$ group of chitosan in weak acidic conditions, the surface of chitosan-capped ZnS QDs possesses positive charges, which is supported by the zeta potential data of the QDs suspension (Fig. S1A $\dagger$ ). The zeta potential of ZnS QDs was measured to be $40.4 \mathrm{mV}$, which indicates that the surface of ZnS QDs is strongly positivecharged. The QDs suspension can be effectively stabilized against aggregation via electrostatic repulsion against van der Waals attraction.

In order to identify the conjugation mode between ZnS QDs and chitosan, FT-IR spectroscopy was applied to this study. The FT-IR spectra of pure chitosan (A) and chitosan-capped ZnS QDs (B) are shown in Fig. 1, and the major characteristic peaks observed in both spectra are shown in Table S1. $\dagger$ The similarity in both the spectral characteristics and the major peak positions indicates that chitosan was well chemically bound onto the surface of the ZnS QDs. Moreover, by discerning the fine differences between the two spectra, the conjugation mode of chitosan and ZnS QDs can be confirmed. It is important to note that the peak around $3300-3500 \mathrm{~cm}^{-1}$, corresponding to stretching vibrations of hydroxyl, amino and amide groups, moved to a lower wavenumber and became broader and stronger, which represents the strong interaction between these groups and ZnS QDs. The mechanism of this interaction may be mainly due to hydrogen and coordinate bonding, as the $\mathrm{ZnS}$ QDs growing in aqueous solution have a large amount of $\mathrm{H}_{2} \mathrm{O}$ and excess $\mathrm{Zn}^{2+}$ bound to the surface of the nanocrystallite, which interact with the $-\mathrm{OH}$ and $-\mathrm{NH}_{2}$ of chitosan via hydrogen

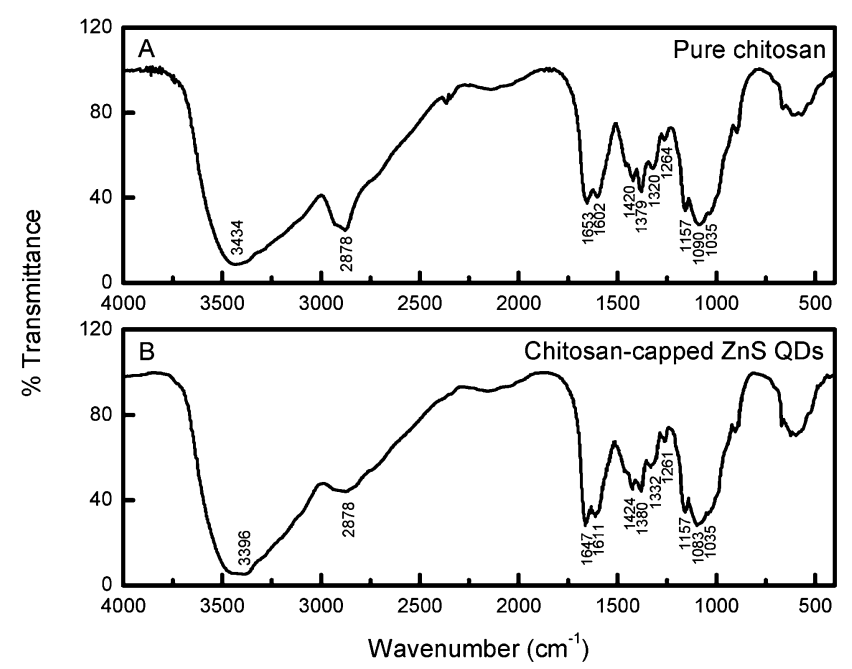

Fig. 1 FT-IR spectra of pure chitosan (A) and chitosan-capped ZnS QDs (B). 
and coordinate bonding. This interaction will result in some other fine spectral changes of covalent bonds in chitosan, especially for those corresponding to $\mathrm{Zn}^{2+}$-bonded atoms. Generally, coordination to metallic ions will reduce the electron density of metallic ion-bonded atoms, which makes stretching require less energy, and increase steric hindrance of covalent bonds corresponding to metallic ion-bonded atoms, which makes bending require more energy. Therefore, the coordination between $\mathrm{Zn}^{2+}$ and chitosan caused the stretching vibration peaks of covalent bonds corresponding to $\mathrm{Zn}^{2+}$-bonded atoms to shift to lower wavenumbers and their bending vibration peaks to move to higher wavenumbers. In Fig. 1, the peaks located at 1264 and $1090 \mathrm{~cm}^{-1}$, respectively assigned to $\mathrm{C}_{2}-\mathrm{H}$ and $\mathrm{C}_{3}-\mathrm{O}$ stretching, shifted to lower wavenumbers of 1261 and $1083 \mathrm{~cm}^{-1}$, and the peak located at $1602 \mathrm{~cm}^{-1}$ assigned to $\mathrm{N}-\mathrm{H}$ bending moved to a higher wavenumber of $1611 \mathrm{~cm}^{-1}$, while the peak located at $1035 \mathrm{~cm}^{-1}$ attributed to $\mathrm{C}_{6}-\mathrm{O}$ stretching
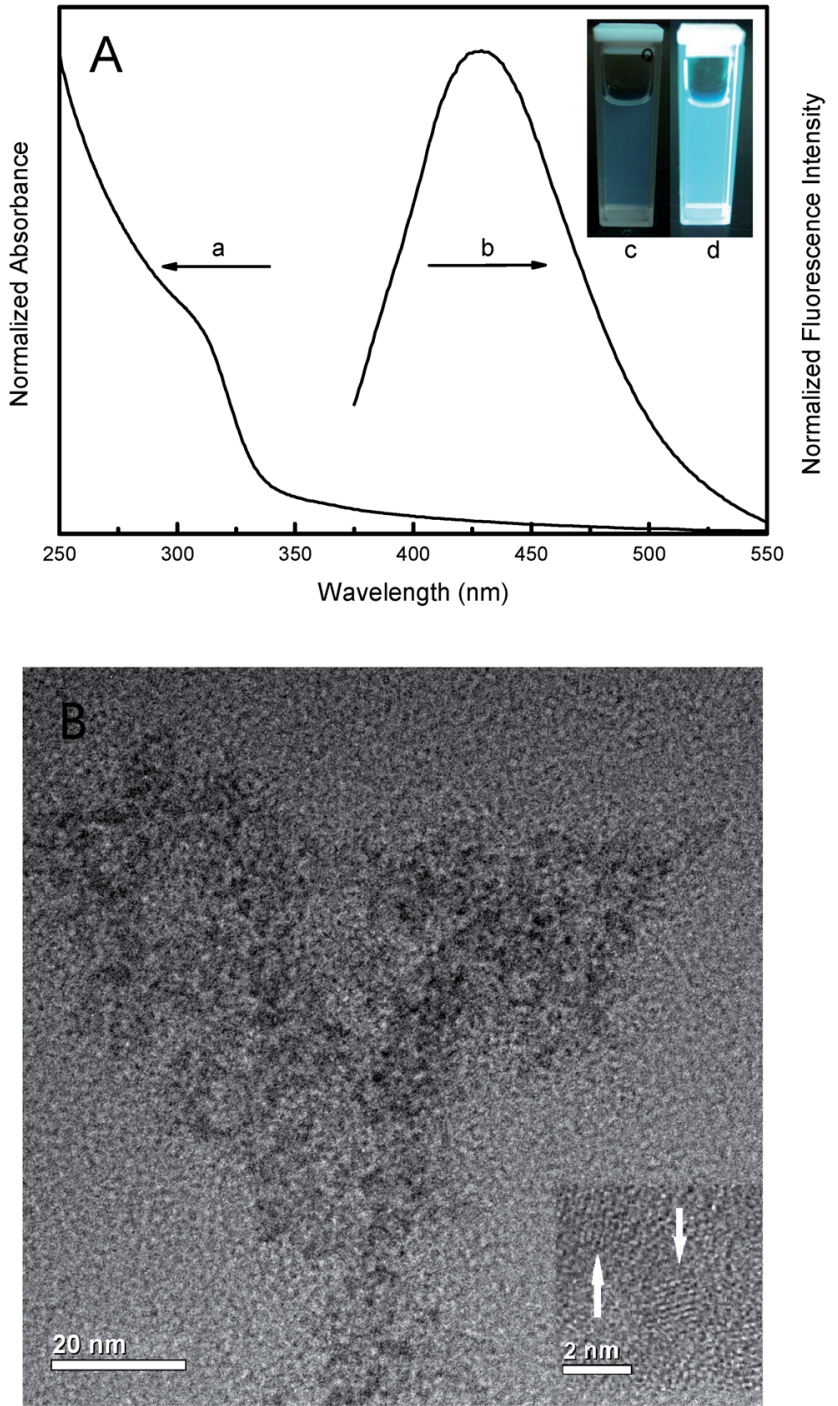

Fig. 2 (A) The absorption spectrum (a) and fluorescence emission spectrum (b) of the as-prepared ZnS QDs, and the digital photographs of the ZnS QDs solution under daylight (c) and a $308 \mathrm{~nm}$ UV lamp (d). (B) HRTEM images of the ZnS QDs. remained stable. These changes show that $\mathrm{N}_{2}$ and $\mathrm{O}_{3}$ of the chitosan molecule are more responsible for the interaction with ZnS QDs and $\mathrm{O}_{6}$ takes little part in the interaction. Other peak changes in Table S1† also confirm the above inference. Since the growth of ZnS QDs is almost in situ, we may deduce that the complex sites between chitosan and $\mathrm{ZnS}$ QDs are mainly located at $\mathrm{C}_{2}$ and $\mathrm{C}_{3}$ of the chitosan repeating units. The inferred formation process of chitosan-capped $\mathrm{ZnS}$ QDs is shown in Scheme 1C.

The chitosan-capped ZnS QDs are optically characterized by UV-vis absorption spectroscopy and fluorometry. The normalized absorption (a) and fluorescence emission (b) spectra are shown in Fig. 2A. The absorption edge of ZnS QDs is at around $310 \mathrm{~nm}$ with a considerable blue-shift compared to that of bulk $\mathrm{ZnS}$ at $340 \mathrm{~nm},{ }^{28}$ showing an apparent quantum confinement effect. The emission maxima centered at $430 \mathrm{~nm}$ with excitation at $315 \mathrm{~nm}$. Photographs of the ZnS QDs solution under daylight and a $308 \mathrm{~nm}$ UV lamp are respectively shown as c and d in Fig. 2A. Under a $308 \mathrm{~nm}$ UV lamp, the QDs solution displayed bright blue fluorescence which was attributed to the defect-related emission of $\mathrm{ZnS}$. The fluorescence spectrum band is relatively narrow and symmetric with full widths at half-maximum (FWHM) of about $85 \mathrm{~nm}$, which reveals that the as-prepared QDs possess fairly uniform particle size due to their relatively low size distribution. ${ }^{29}$ The molar extinction coefficient $(\varepsilon)$ of the chitosan-capped ZnS QDs at $315 \mathrm{~nm}$ was estimated to be about $3.3 \times 10^{5} \mathrm{M}^{-1} \mathrm{~cm}^{-1},{ }^{27}$ thus the molar concentration of the $\mathrm{ZnS}$ QDs was calculated to be nearly $3.5 \times 10^{-6} \mathrm{~mol} \mathrm{~L}^{-1}$ according to the Beer-Lambert law. HRTEM was also performed to study the morphology of the as-prepared chitosancapped ZnS QDs. Fig. 2B shows a typical image of the obtained ZnS QDs. The shape of these nanoparticles was close to spherical and partly aggregated, with diameters ranging from 1.5 to $2.0 \mathrm{~nm}$. The average particle size was about $1.8 \mathrm{~nm}$.

The fluorescence quantum yield (QY) represents the efficiency of a fluorescent material in converting the excitation into fluorescent emission. According to Williams' method, ${ }^{30}$ by using $\mathrm{Rh} 6 \mathrm{G}$ ( $\mathrm{QY}=95 \%$, in ethanol) as the reference and exciting all the samples at $315 \mathrm{~nm}$, the QY of chitosan-capped ZnS QDs was calculated from the following equation:

$$
\mathrm{QY}_{\mathrm{ZnS}}=\mathrm{QY}_{\mathrm{Rh} 6 \mathrm{G}} \frac{m_{\mathrm{ZnS}}}{m_{\mathrm{Rh} 6 \mathrm{G}}}\left(\frac{\eta_{\text {water }}}{\eta_{\text {ethanol }}}\right)^{2}
$$

where $m$ and $\eta$ are the slope of the integrated fluorescence intensity versus absorbance at $315 \mathrm{~nm}$ and the refractive index of the solvent respectively. The integrated fluorescence intensity was obtained by integrating the emission intensity over the entire wavelength range under the emission peak, and the absorbance was kept between 0.01 and 0.1 to avoid the selfabsorption effect. With the QY of Rh $6 \mathrm{G}$ taken as $95 \%$, we obtained the QY of the ZnS QDs as $11.8 \%$. Since it has been widely accepted that QDs whose QY is more than $10 \%$ can be considered for practical applications, ${ }^{31}$ it could be concluded that the obtained ZnS QDs can show a satisfactory fluorescent analytical performance in this assay. 


\subsection{Effects of BPA on the fluorescence of chitosan-capped ZnS QDs}

According to the analytical procedure introduced in Section 2.3, the fluorescence emission spectra of the as-prepared chitosancapped ZnS QDs (Fig. 3) in the absence (a) and presence (b) of BPA were recorded. The fluorescence band of chitosan-capped ZnS QDs was centered at $430 \mathrm{~nm}$. When BPA was added to the ZnS QDs solution, significant quenching of fluorescence intensity was observed. In order to identify the origins of the fluorescence quenching, control experiments were performed. Free $\mathrm{ZnCl}_{2}$, chitosan and $\mathrm{Zn}^{2+}$-chitosan complex with the same concentrations as those used in the synthesis of ZnS QDs were added into the mixture solution of ZnS QDs and BPA. The obtained results revealed that these substances had no contribution to the quenching effect of BPA on the fluorescence emission of ZnS QDs. Thus, the quenching effect was attributed to the interaction between BPA and chitosan-capped ZnS QDs. Considering this remarkable quenching of fluorescence intensity, the possibility of developing a simple and sensitive fluorescence chemosensor for rapid detection of BPA should be further studied.

\subsection{Optimization of assay conditions}

The influence of reaction time on the fluorescence intensity of the system was investigated at room temperature. The optimum incubation time for the reaction between ZnS QDs and BPA to reach equilibrium was examined by recording the fluorescence spectra every $0.5 \mathrm{~min}$ in real time (Fig. $4 \mathrm{~A}$ ). The results show that after addition of BPA into the $\mathrm{ZnS}$ QDs solution the fluorescence intensity decreased by prolonging the reaction time and the equilibrium was obtained within $4.0 \mathrm{~min}$. $F_{0} / F$ was nearly constant after $4.0 \mathrm{~min}$ and the fluorescence intensity of the BPA-ZnS QDs system could remain stable for at least $50 \mathrm{~min}$. Thus the reaction time was fixed at $4.0 \mathrm{~min}$.

It is well known that the fluorescence intensity of QDs is greatly affected by the medium's pH. Using $0.1 \mathrm{~mol} \mathrm{~L}^{-1} \mathrm{NaOH}$ or $0.1 \mathrm{~mol} \mathrm{~L}^{-1} \mathrm{HCl}$ for $\mathrm{pH}$ adjustment, the effects of the solution

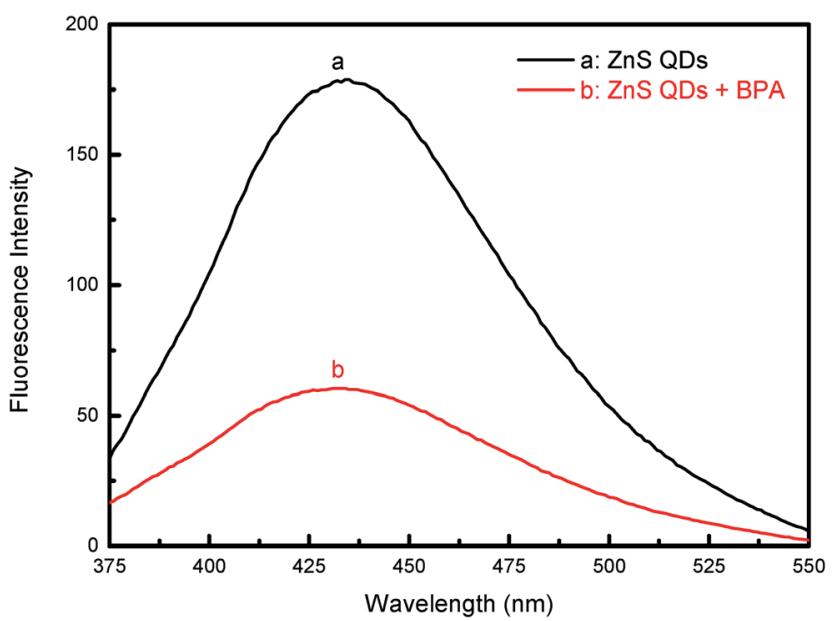

Fig. 3 The fluorescence emission spectra of $1.2 \times 10^{-6} \mathrm{~mol} \mathrm{~L}^{-1} \mathrm{ZnS}$ QDs in the absence (a) and presence (b) of $150 \mu \mathrm{g} \mathrm{L}^{-1}$ BPA.
$\mathrm{pH}$ on the fluorescence intensity of ZnS QDs in the absence and presence of BPA were investigated and the results are shown in Fig. $4 \mathrm{~B}$ and $4 \mathrm{C}$ respectively. In Fig. $4 \mathrm{~B}$, it can be seen that stable and high fluorescence intensity was obtained in the $\mathrm{pH}$ range of 4.0-4.5 and $\mathrm{pH}$ lower than 4.0 or higher than 4.5 resulted in a significant decrease of fluorescence intensity. Under the condition of $\mathrm{pH}<4.0$, the low fluorescence intensity is the result of dissociation of the QDs, as the interaction between chitosan and ZnS QDs was wrecked and the stability of the QDs dramatically decreased. With an increase in $\mathrm{pH}$, deprotonation of the $-\mathrm{NH}_{2}$ group in the chitosan molecule occurred. The deprotonation could strengthen the interaction between chitosan and ZnS QDs to some degree, which brought about fluorescence enhancing to some extent. However, the fluorescence intensity began to decrease with a further increase of $\mathrm{pH}$ above 4.5 , as the solubility of chitosan decreased, which resulted in partial precipitation of the QDs. In the presence of BPA, as illustrated in Fig. $4 \mathrm{C}, F_{0} / F$ is also stable and high in the $\mathrm{pH}$ range of 4.0-4.5. Thus, the optimal $\mathrm{pH}$ was chosen to be 4.0 for further experiments.

To gain the widest linear range and the highest sensitivity of the calibration function, the effect of $\mathrm{ZnS}$ QDs concentration on the $F_{0} / F$ of the BPA-ZnS QDs system was further investigated. A relatively low concentration of ZnS QDs can correspondingly only provide very weak fluorescence emission, which is not beneficial for obtaining a wider linear range. With an increase of the ZnS QDs concentration, the fluorescence intensity of the system increases gradually (not shown). A very high QDs concentration could enlarge the linear range but at the expense of sensitivity. As shown in Fig. $4 \mathrm{D}$, the maximum $F_{0} / F$ was achieved when the concentration was $1.2 \times 10^{-6} \mathrm{~mol} \mathrm{~L}^{-1}$. Thus, after comprehensively considering high sensitivity and wide linear range, a QDs concentration of $1.2 \times 10^{-6} \mathrm{~mol} \mathrm{~L}^{-1}$ (in the test sample) is recommended for further research.

\subsection{Calibration and sensitivity}

As shown in Fig. 5, under the optimal conditions mentioned above, the addition of BPA gradually decreased the fluorescence intensity of the ZnS QDs, so a quantitative determination of BPA based on fluorescence quenching was possible. The inset in Fig. 5 illustrates that $F_{0} / F$ exhibits a good linear relationship with the concentration of BPA over a wide range from 0.05 to $300 \mu \mathrm{g} \mathrm{\textrm {L } ^ { - 1 }}$, which is well described by a Stern-Volmer (SV) equation with a determination coefficient of 0.99531 :

$$
\frac{F_{0}}{F}=0.97064+0.01431 c_{\mathrm{BPA}}
$$

where $c_{\mathrm{BPA}}$ is the concentration of BPA $\left(\mu \mathrm{g} \mathrm{\textrm {L } ^ { - 1 }}\right)$. The limit of detection (LOD) for BPA in water is $0.08 \mu \mathrm{g} \mathrm{L}^{-1}$ with the ratio of signal to noise of $3(S / N=3)$. The relative standard deviation (RSD) for six parallel determinations of a solution containing $25.0 \mu \mathrm{g} \mathrm{L}^{-1}$ BPA was $1.2 \%$. This indicates that the method can offer good precision for the detection of BPA. Compared with the existing methods (shown in Table $\mathbf{S} 2 \dagger$ ), a relatively low detection limit was obtained and it was also much lower than $30.0 \mu \mathrm{g} \mathrm{L}{ }^{-1}$, which is the LOD given by the HPLC method according to Chinese National Standard GB/T 23296.16-2009 

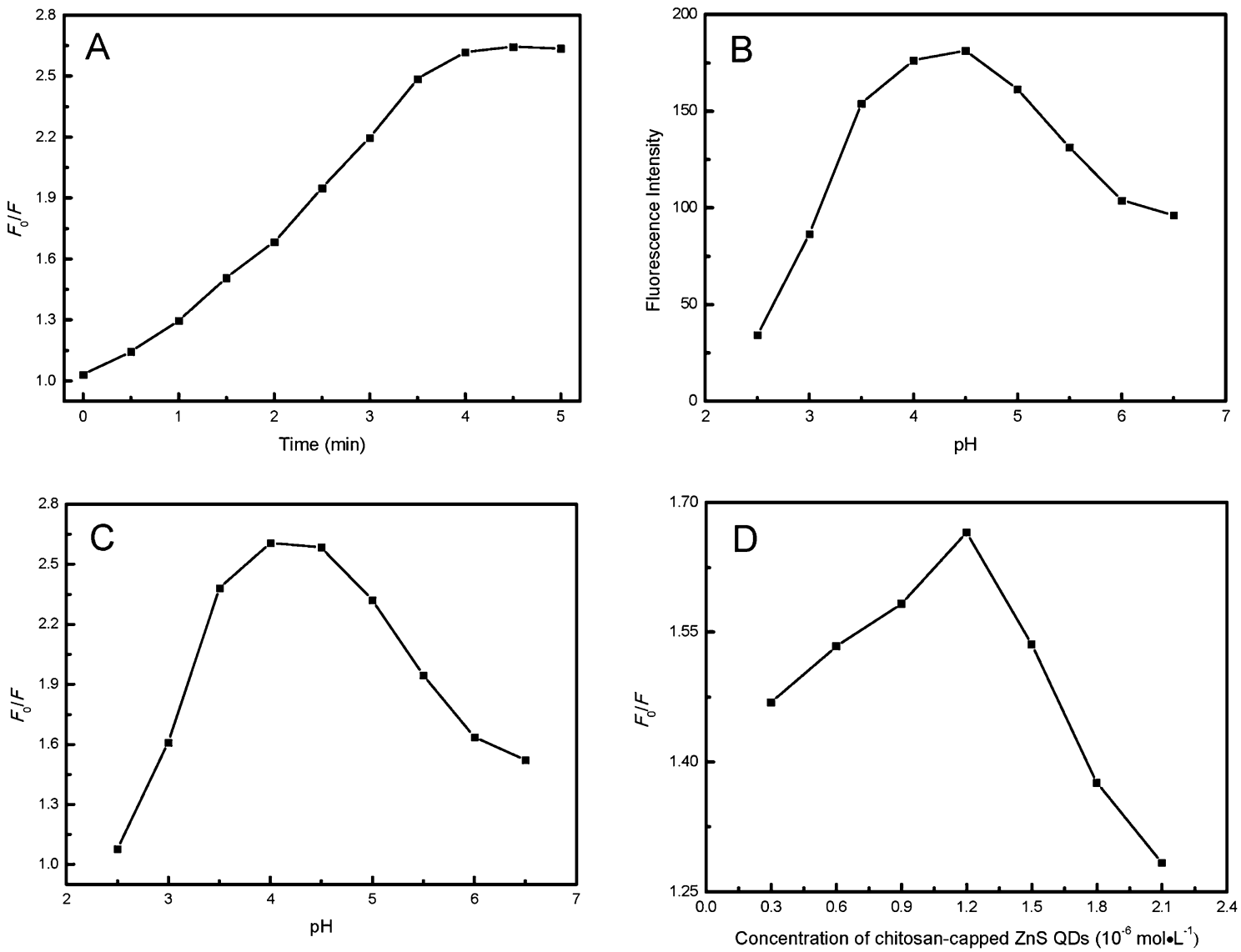

Fig. 4 (A) Variation of $F_{0} / F$ versus the reaction time for $Z n S Q D s$ after addition of BPA. (B) Effect of pH on the fluorescence intensity of ZnS QDs. (C) Effect of $\mathrm{pH}$ on $F_{0} / F$ with the presence of BPA. (D) Effect of ZnS QDs concentration on $F_{0} / F$ with the presence of BPA. Concentrations: ZnS QDs for $A, B$ and $C, 1.2 \times 10^{-6} \mathrm{~mol} \mathrm{~L}^{-1}$; BPA for $A$ and $C, 150 \mu \mathrm{g} \mathrm{L}^{-1}$, for $\mathrm{D}, 50 \mu \mathrm{g} \mathrm{L}^{-1}$.

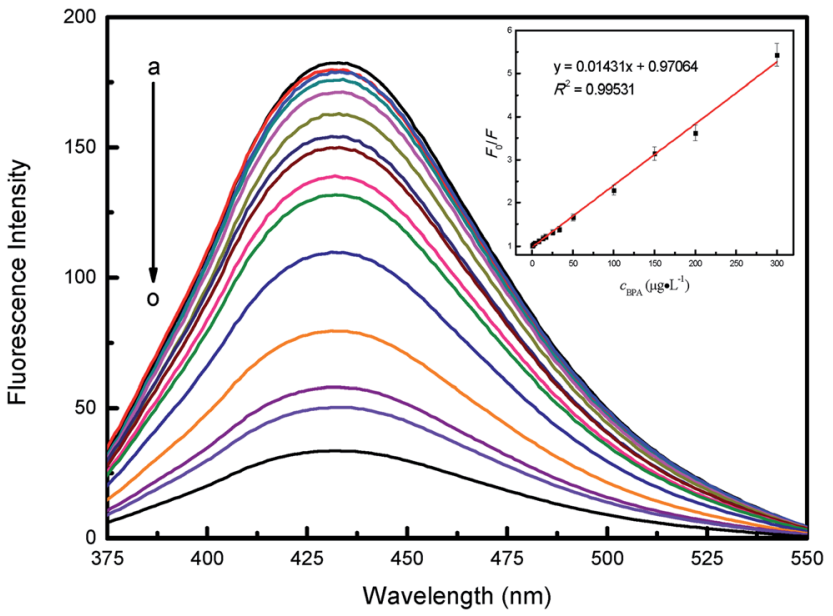

Fig. 5 Fluorescence emission spectra of ZnS QDs in the presence of increasing concentrations of BPA. The concentration of BPA in samples (a)-(o) was $0,0.50,0.83,1.66,3.33,8.33,13.3,16.6,25.0,33.3$, $50.0,100,150,200$ and $300 \mu \mathrm{g} \mathrm{L}^{-1}$, respectively. The concentration of ZnS QDs was $1.2 \times 10^{-6} \mathrm{~mol} \mathrm{~L}^{-1}$. The inset shows the calibration curve of $F_{0} / F$ versus the concentration of BPA.

(Food contact materials - Polymer: Determination of bisphenol A in food simulants-High performance liquid chromatography). There are obvious advantages of the proposed method, such as easy sample pretreatment, short analysis time, low cost and nontoxicity.

\subsection{Interference studies}

The fluorescence titration of chitosan-capped ZnS QDs with various coexisting substances was performed to examine the detection selectivity. Table $\mathrm{S} 3 \dagger$ describes the influence of coexisting substances on the fluorescence intensity of ZnS QDs with BPA. For coexisting substances, $\mathrm{K}^{+}, \mathrm{Na}^{+}, \mathrm{Al}^{3+}, \mathrm{Ca}^{2+}, \mathrm{Ba}^{2+}, \mathrm{NH}_{4}^{+}$, $\mathrm{Mg}^{2+}, \mathrm{CO}_{3}{ }^{2-}, \mathrm{SO}_{4}{ }^{2-}, \mathrm{PO}_{4}{ }^{3-}, \mathrm{CH}_{3} \mathrm{COO}^{-}, \mathrm{NO}_{3}{ }^{-}, \mathrm{Cl}^{-}, \mathrm{Mn}^{2+}$, glucose, lactose and glycine induced less than $\pm 5 \%$ change in the fluorescence intensity of ZnS QDs and the tolerance limits of

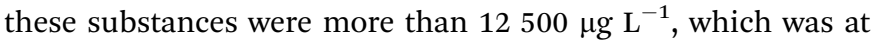
least 500 times the coexisting BPA concentration and which means that these coexisting substances had no interference for detection of $25.0 \mu \mathrm{g} \quad \mathrm{L}^{-1}$ BPA. $\mathrm{Zn}^{2+}$ had a fluorescence enhancement effect at a relatively higher concentration since they were adsorbed onto the surface of $\mathrm{ZnS}$ QDs and the increase of $S$ surface vacancies improved the defect-related emission of $\mathrm{ZnS}$ QDs to some extent. $\mathrm{Fe}^{3+}, \mathrm{Cu}^{2+}, \mathrm{Hg}^{2+}$ and $\mathrm{Ag}^{+}$ exhibited an effective quenching effect, which was attributed to their strong chemical adsorption onto the ZnS QDs surface. However, the tolerance limits of these ions were at least 5 times that of the BPA concentration and much higher than their 
potential concentration in real samples. Thus, the detecting system described here possesses a good selective fluorescence response toward BPA and it could be applied to determine BPA in real samples.

\subsection{Detection of BPA in real samples}

The proposed method was applied to the determination of BPA in tap, rain and packaged drinking water. The method of standard additions was used to evaluate the analytical performance of the fluorescence sensor and the results are shown in Table 1. It can be seen that the recoveries were in the range of 95.9-105.8\% with RSD values between $1.5 \%$ and $4.0 \%$, indicating that the fluorescence sensor might be sufficient and satisfactory for BPA detection in these water samples. No BPA was detected in the tap water sample. From one packaged drinking water sample, BPA was detected at a very low amount of $0.19 \mu \mathrm{g} \mathrm{L}^{-1}$, and for the other, it was not detected. This very low amount of BPA in packaged drinking water may be caused by BPA migration from the packages under unreasonable storage conditions. $0.41 \mu \mathrm{g} \mathrm{L}^{-1}$ BPA was detected in the rain water sample, which may be due to a very slow BPA migration from domestic garbage and wastewater to the global water cycle.

Samples of plastic cups, feeding bottles, microwave lunch boxes and epoxy resin-based bowls with different brands were also inspected. Various ultrasonic and microwave extraction conditions were investigated. The optimal conditions selected were $90{ }^{\circ} \mathrm{C}$ and $90 \mathrm{~min}$ for ultrasonic extraction, and $10 \mathrm{~min}$ for microwave extraction, since under these conditions the extraction periods were significantly shortened and the extraction effect were equivalent to those under the condition stipulated by GB/T 23296.1-2009. After extraction procedures under these optimal conditions, leaching solutions of the plastic samples were examined using the proposed method and the results are also shown in Table 1. High recoveries from $96.4 \%$ to $104.3 \%$ were obtained for these plastic samples with RSD values between $1.0 \%$ and $4.4 \%$. The result demonstrates that the proposed analytical method was also competent for BPA detection of plastic samples. It can be seen that no BPA was detected in the samples of the polypropylene (PP)-made plastic

Table 1 Concentrations and recoveries of BPA in various water samples and plastic leaching solutions

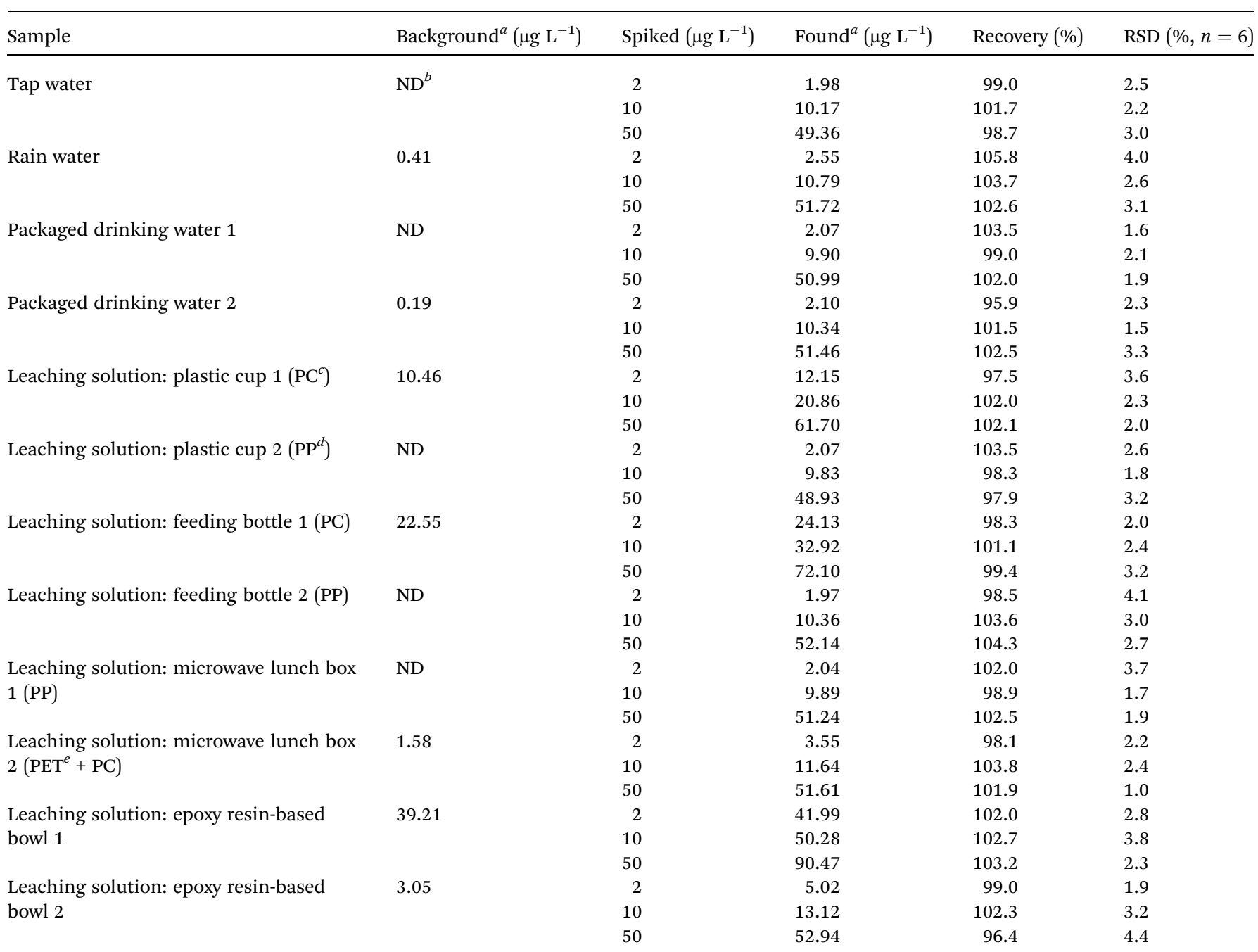

${ }^{a}$ Mean of six measurements. ${ }^{b}$ Not detected. ${ }^{c}$ PC: polycarbonate. ${ }^{d}$ PP: polypropylene. ${ }^{e}$ PET: polyethylene terephthalate. 
products. However, in some PC-made or PC-containing materials, various amounts of BPA could be detected, which indicates that the employed BPA extraction processes in this assay are very effective.

\subsection{Quenching mechanism}

Up to now, many quenching mechanisms including energy transfer, charge diverting and surface absorption have been proposed to explain the fluorescence quenching phenomena of QDs. To explore the possible mechanism involved in the interaction between chitosan-capped ZnS QDs and BPA, the UV-vis absorption spectra of BPA, ZnS QDs and ZnS QDs with added BPA were investigated. Shown as curve a in Fig. S2, $\uparrow$ BPA had no absorption in the wavelength range of $300-600 \mathrm{~nm}$, so the quenching effect of BPA on the fluorescence of ZnS QDs is not due to an inner filter effect resulting from the absorption of the emission wavelength by BPA. No obvious change was observed for the ZnS QDs absorption spectra before and after adding BPA (curve b and $c$ in Fig. $\mathrm{S} 2 \dagger$ ) and no perceptible red shift on the maximum emission wavelength was found in Fig. 5 with increasing concentration of BPA, which indicates that the presence of BPA mainly influences the surface status and not the size of the ZnS QDs. Generally, in an alkaline environment, the complexation reaction between $\mathrm{BPA}$ and $\mathrm{Zn}^{2+}$ on the $\mathrm{ZnS}$ QDs surface may occur. As the current ZnS QDs-BPA system is weakly acidic, there is little probability of the complexation reaction. The zeta potential of BPA in the weakly acidic solution was also measured to be $23.0 \mathrm{mV}$ (Fig. S1B $\dagger$ ). Since both BPA and ZnS QDs possessed positive charges, there was no electrostatic attractive interaction between them. Thus, the quenching may be due to the hydrogen bonding between BPA and chitosan on the surface of the QDs (Scheme 1D). BPA could bind to the surface of chitosan-capped ZnS QDs like a cap through the hydrogen-bond interaction between the $-\mathrm{OH}$ of BPA and the $-\mathrm{NH}_{2} /-\mathrm{OH}$ of chitosan. ${ }^{32}$ The hydrogen bonds lead chitosan molecules to partly peel off from the surface of the ZnS QDs, and then the surface changes induced the fluorescence quenching of the ZnS QDs. ${ }^{21}$

\section{Conclusion}

In summary, with chitosan as a capping and stabilizing agent, water-soluble ZnS QDs were easily prepared. By using the nontoxic ZnS QDs as a fluorescence probe, a novel and ecofriendly technique for rapid BPA detection with high sensitivity and selectivity was developed based on the quenching effect of BPA on the fluorescence emission of ZnS QDs. The possible quenching mechanism is due to the surface change of ZnS QDs, induced by hydrogen bonding between BPA and chitosan on the surface of ZnS QDs. The factors affecting both the synthesis of chitosan-capped ZnS QDs and the fluorescence detection for BPA were also examined. Under the optimal assay conditions, a relatively wide linear range (from 0.50 to $300 \mu \mathrm{g} \mathrm{L}^{-1}$ ) and low detection limit $\left(0.08 \mu \mathrm{g} \mathrm{L}^{-1}\right)$ were obtained. Most of the common coexisting substances hardly interfered with the determination of BPA. The proposed method was successfully applied to the recognition of BPA in water and plastic samples, and simplicity, rapidity, high sensitivity and low cost was exhibited in the detection process. Thus, it appears to be a promising candidate for on-site rapid screening of BPA contamination in water and plastic samples.

\section{Acknowledgements}

This work was financially supported by the National Natural Science Foundation of China (no. 20905031), the Natural Science Foundation of Jilin Province (no. 201215024) and Innovation Projects of Science Frontiers and Interdisciplinary of Jilin University.

\section{References}

1 C. A. Staples, K. Woodburn, N. Caspers, A. T. Hall and G. M. Klecka, Hum. Ecol. Risk Assess., 2002, 8, 1083-1105.

2 D. A. Crain, M. Eriksen, T. Iguchi, S. Jobling, H. Laufer, G. A. LeBlanc and L. J. Guillette. Jr, Reprod. Toxicol., 2007, 24, 225-239.

3 L. N. Vandenberg, R. Hauser, M. Marcus, N. Olea and W. V. Welshons, Reprod. Toxicol., 2007, 24, 139-177.

4 N. K. Wilson, J. C. Chuang, M. K. Morgan, R. A. Lordo and L. S. Sheldon, Environ. Res., 2007, 103, 9-20.

5 H. H. Le, E. M. Carlson, J. P. Chua and S. M. Belcher, Toxicol. Lett., 2008, 176, 149-156.

6 E. Carlsen, A. Giwercman, N. Keiding and N. E. Skakkebæk, Environ. Health Perspect., 1995, 103, 137-139.

7 X. H. Long, R. Steinmetz, N. Ben-Jonathan, A. Caperell-Grant, P. C. M. Young, K. P. Nephew and R. M. Bigsby, Environ. Health Perspect., 2000, 108, 243-247.

8 X. W. Tan, Y. X. Song, R. P. Wei and G. Y. Yi, Chin. J. Anal. Chem., 2012, 40, 1409-1414.

9 A. Motoyama, A. Suzuki, O. Shirota and R. Namba, Rapid Commun. Mass Spectrom., 1999, 13, 2204-2208.

10 M. K. R. Mudiam, R. Jain, V. K. Dua, A. K. Singh, V. P. Sharma and R. C. Murthy, Anal. Bioanal. Chem., 2011, 401, 16951701.

11 Y. Lu, J. R. Peterson, J. J. Gooding and N. A. Lee, Anal. Bioanal. Chem., 2012, 403, 1607-1618.

12 S. X. Zhong, S. N. Tan, L. Y. Ge, W. P. Wang and J. R. Chen, Talanta, 2011, 85, 488-492.

13 C. M. Yu, L. L. Gou, X. H. Zhou, N. Bao and H. Y. Gu, Electrochim. Acta, 2011, 56, 9056-9063.

14 C. Lu, J. G. Li, Y. Yang and J. M. Lin, Talanta, 2010, 82, 15761580.

15 G. F. Li, S. Morita, S. Ye, M. Tanaka and M. Osawa, Anal. Chem., 2004, 76, 788-795.

16 Z. L. Mei, H. Q. Chu, W. Chen, F. Xue, J. Liu, H. N. Xu, R. Zhang and L. Zheng, Biosens. Bioelectron., 2013, 39, 26-30.

17 K. Hegnerová, M. Piliarik, M. Šteinbachová, Z. Flegelová, H. Černohorská and J. Homola, Anal. Bioanal. Chem., 2010, 398, 1963-1966.

18 X. Y. Liu, C. Nakamura, I. Tanimoto, S. Miyake, N. Nakamura, T. Hirano and J. Miyake, Anal. Chim. Acta, 2006, 578, 43-49. 
19 J. Fan, H. Q. Guo, G. G. Liu and P. G. Peng, Anal. Chim. Acta, 2007, 585, 134-138.

20 Y. Kim, J. B. Jeon and J. Y. Chang, J. Mater. Chem., 2012, 22, 24075-24080.

21 R. Kuang, X. Kuang, S. Y. Pan, X. D. Zheng, J. C. Duan and Y. Q. Duan, Microchim. Acta, 2010, 169, 109-115.

22 X. Wang, H. L. Zeng, Y. L. Wei and J. M. Lin, Sens. Actuators, $B, 2006,114,565-572$.

23 S. A. O. Gomes, C. S. Vieira, D. B. Almeida, J. R. SantosMallet, R. F. S. Menna-Barreto, C. L. Cesar and D. Feder, Sensors, 2011, 11, 11664-11678.

24 H. Li, M. Y. Li, W. Y. Shih, P. I. Lelkes and W. H. Shih, J. Nanosci. Nanotechnol., 2011, 11, 3543-3551.

25 Z. Li, Y. M. Du, Z. L. Zhang and D. W. Pang, React. Funct. Polym., 2003, 55, 35-43.
26 M. Sharma, S. Singh and O. P. Pandey, J. Appl. Phys., 2010, 107, 104319.

27 A. Jaiswal, P. Sanpui, A. Chattopadhyay and S. S. Ghosh, Plasmonics, 2011, 6, 125-132.

28 J. Q. Zhuang, X. D. Zhang, G. Wang, D. M. Li, W. S. Yang and T. J. Li, J. Mater. Chem., 2003, 13, 1853-1857.

29 S. Chowdhury, A. M. P. Hussain, G. A. Ahmed, F. Singh, D. K. Avasthi and A. Choudhury, Mater. Res. Bull., 2008, 43, 3495-3505.

30 A. T. R. Williams, S. A. Winfield and J. M. Miller, Analyst, 1983, 108, 1067-1071.

31 C. H. Song, Y. T. Li, J. Li, Y. J. Wei, Y. Z. Hu and Y. Wei, Spectrosc. Spectral Anal., 2008, 28, 169-173.

32 T. I. Chanu and D. P. S. Negi, Chem. Phys. Lett., 2010, 491, 7579. 